\title{
Cluster based routing protocol for mobile nodes in wireless sensor network
}

\begin{abstract}
Mobility of sensor nodes in wireless sensor network (WSN) has posed new challenges particularly in packet delivery ratio and energy consumption. Some real applications impose combined environments of fixed and mobile sensor nodes in the same network, while others demand a complete mobile sensors environment. Packet loss that occurs due to mobility of the sensor nodes is one of the main challenges which comes in parallel with energy consumption. In this paper, we use cross layer design between medium access control (MAC) and network layers to overcome these challenges. Thus, a cluster based routing protocol for mobile sensor nodes (CBR-Mobile) is proposed. The CBR-Mobile is mobility and traffic adaptive protocol. The timeslots assigned to the mobile sensor nodes that had moved out of the cluster or have not data to send will be reassigned to incoming sensor nodes within the cluster region. The protocol introduces two simple databases to achieve the mobility and traffic adaptively. The proposed protocol sends data to cluster heads in an efficient manner based on received signal strength. In CBR-Mobile protocol, cluster based routing collaborates with hybrid MAC protocol to support mobility of sensor nodes. Schedule timeslots are used to send the data message while the contention timeslots are used to send join registration messages. The performance of proposed CBR-Mobile protocol is evaluated using MATLAB and was observed that the proposed protocol improves the packet delivery ratio, energy consumption, delay and fairness in mobility environment compared to LEACH-Mobile and AODV protocols.
\end{abstract}

Keyword: Cluster head; LEACH; LEACH-Mobile; Mobility; WSN 